Poisson regression analysis, and their $95 \%$ confidence interval rates were estimated.

The characteristics of the patients enrolled in both studies were compared. After the intervention, the trend was to attend patients with more severe conditions: higher frequency of liver failure, chronic obstructive lung disease, higher proportion of dirty surgical wounds, and higher scores on both Study on the Efficacy of Nosocomial Infection Control (SENIC) and National Nosocomial Infections Surveillance System indices. There were no significant differences in emergency surgery, duration of surgery, age, and gender. After the intervention, unnecessary chemoprophylaxis was reduced drastically, and a significant reduction in preoperative stay was observed. The nosocomial incidence rate fell from 18.4 to 14 per 1,000 patient-days. This reduction yielded an incidence rate ratio of 0.56 (95\% confidence interval, $0.43 \%-0.74 \%$ ), adjusted for several variables (SENIC index, serum creatinine level, serum albumin level, antihistamine $\mathrm{H}_{2}$ level, surgical wound, body mass index, chemoprophylaxis, and community-acquired infection). Significant reductions in surgical-site infection and urinary tract infection were observed, but the rate of respiratory tract infection remained unchanged.

The authors concluded that surveillance was effective in reducing NI.

FROM: Delgado-Rodriguez M, Gomez-Ortega A, Sillero-Arenas M, Martinez-Gallego G, Medina-Cuadros M, Llorca J. Efficacy of surveillance in nosocomial infection control in a surgical service. Am J Infect Control 2001;29:289-294.

\section{Non-Touch Sink Taps Possible Source of Bacteria}

Halabi and colleagues recently published a study that pointed out that non-touch fittings are associated with bacterial contamination. Hospitals and other healthcare facilities have begun to install touchless water taps to lower water consumption as a cost-saving measure and to prevent healthcare workers from touching the tap, thus promoting hygiene. This study analyzed the bacteriological water quality of 38 non-touch water taps in different settings in a 450-bed secondary-care hospital in Upper Austria. Two different tap types were installed: 23 taps were without temperature selection, and 15 were with temperature selection (cold and warm). A membrane filtration method was used for bacteriologic assays, and the authors screened for both indicator organisms and Pseudomonas aeruginosa in $100-\mathrm{mL}$ water samples. In 10 non-touch taps without temperature selection, the authors also screened for Legionella species in $500 \mathrm{~mL}$ water samples.

Seventy-four percent of the taps without temperature selection and $7 \%$ of the taps with temperature selection showed contamination with $P$ aeruginosa $(P<.001)$. None of the taps showed contamination with indicator organisms. Detailed analysis of the source of contamina- tion revealed that the magnetic valve and the outlet itself were heavily contaminated, whereas the junction from the central pipe system was free of contamination. All 10 analyzed taps showed contamination with Legionella species. The authors concluded that the local contamination of non-touch fittings is a result of the low amount of water that flows through the outlet, the low water pressure, and the column of water, which is "still-standing" and has a temperature of approximately $35^{\circ} \mathrm{C}$, thus providing nearly ideal growth conditions for $P$ aeruginosa. Additionally, the presence of materials such as rubber and PVC in the fittings enhances the adhesion of $P$ aeruginos $a$ and thus the production of biofilms.

FROM: Halabi M, Wiesholzer-Pittl M, Schoberl J, Mittermayer H. Non-touch fittings in hospitals: a possible source of Pseudomonas aeruginosa and Legionella spp. $J$ Hosp Infect 2001;49:117-121.

\section{Epidemiology of VRE in Liver Transplant Patients}

Vancomycin-resistant enterococci (VRE) are increasingly important as pathogens in liver transplant patients. To guide control efforts, Bakir and coinvestigators, from the University of Chicago Hospitals, conducted an epidemiological study of the frequency, source, and modes of transmission of VRE at their institution. During September 1998 through August 1999, weekly surveillance cultures were obtained from consenting liver transplant patients and from environmental surfaces in their rooms. Pooled handwash specimens from personnel also were obtained. Specimens were processed on selective media to detect VRE, and isolates were typed by pulsed-field gel electrophoresis. Information was collected from patient records concerning in-hospital treatment and clinical course.

Serial cultures were obtained during 33 admissions of 29 patients. VRE were detected in initial specimens from 6 admissions, and nosocomial acquisition of VRE occurred in $12(44 \%)$ of the remaining 27 admissions. Seven different strain types of VRE were detected. The initial site of acquisition was stool in all cases; bile became culture-positive in only 2 patients. Overall, 16 $(55 \%)$ of the 29 patients became colonized, usually after transplantation. VRE were detected in environmental cultures during 10 admissions and in 2 of 21 pooled hand washes. No statistically significant differences in clinical status or treatment were found when colonized patients were compared to non-colonized controls. The only VRE infection resulted from a choledochojejunostomy anastomotic leak.

The authors concluded that alimentary tract colonization by VRE occurred commonly in liver transplant patients, probably by cross-transmission. The clinical consequences were modest in the patients studied, but colonized transplant patients provide a substantial reservoir for continued VRE transmission in hospitals.

FROM: Bakir M, Bova JL, Newell KA, Millis JM, Buell JF, Arnow PM. Epidemiology and clinical conse- 
quences of vancomycin-resistant enterococci in liver transplant patients. Transplantation 2001;72:1032-1037.

\section{VRE Colonization in Hemodialysis Patients}

Vancomycin-resistant enterococci (VRE) are increasing in prevalence at many institutions and are reported often in dialysis patients. Tokars and coinvestigators from the CDC's Hospital Infections Program studied the prevalence of, and risk factors for, VRE at seven outpatient hemodialysis centers (three in Baltimore, Maryland, and four in Richmond, Virginia). Rectal or stool cultures were performed on consenting hemodialysis patients during December 1997 to April 1998. Repeat cultures were obtained from consenting patients during May to July 1998 (median, 120 days later). Clinical and laboratory data and functional status ( 1 to 10 scale: 1 , normal function; 9 , home attendant, not totally disabled; 10 , disabled, living at home) were recorded.

Of 478 cultures performed, 20 (4.2\%) were positive for VRE. Among the seven centers, the prevalence of VREpositive cultures varied from $1.0 \%$ to $7.9 \%$. Independently significant risk factors for a VRE-positive culture were a functional score of 9 to 10 (odds ratio, $6.9 ; P<.001$ ), antimicrobial receipt within 90 days before culture (odds ratio, $6.1 ; P<.001)$, and a history of injection drug use odds ratio, 5.4; $P=.004)$.

The authors concluded that VRE-colonized patients were present at all seven participating centers, suggesting that careful infection control precautions should be used at all centers to limit transmission. In agreement with previous studies, VRE colonization was found more frequently in patients who had received antimicrobial agents recently, underscoring the importance of judicious antimicrobial use in limiting selection for this potential pathogen.

FROM: Tokars JI, Gehr T, Jarvis WR, Anderson J, Armistead N, Miller ER, et al. Vancomycin-resistant enterococci colonization in patients at seven hemodialysis centers. Kidney Int 2001;60:1511-1516.

\section{Community-Onset $S$ aureus Bacteremia}

Morin and Hadler, from the Epidemiology Program, Connecticut Department of Public Health, conducted a study that retrospectively analyzed the magnitude and epidemiology of community-onset Staphylococcus aureus (COSA) infections and methicillin-resistant $S$ aureus (MRSA) infections in four Connecticut metropolitan areas (population, 1.1 million). The study looked at hospital medical records of persons admitted with $S$ aureus bacteremia in 1998. COSA was categorized as "healthcare associated," "with underlying medical condition," or "no underlying medical condition."

Overall, $48 \%$ of $S$ aureus bacteremic infections were COSA (incidence, 17 cases $/ 100,000$ persons). Incidence increased with age and higher population density. In all,
$62 \%$ of infections were healthcare associated; $85 \%$ of the remaining cases had underlying medical conditions. MRSA accounted for $16 \%$ of healthcare-associated cases and cases with underlying conditions, but no cases with no underlying conditions. COSA bacteremic infections are as common as those due to pneumococci. MRSA is a well-established cause of COSA among persons at high medical risk for $S$ aureus infection.

FROM: Morin CA, Hadler JL. Population-based incidence and characteristics of community-onset Staphylococcus aureus infections with bacteremia in 4 metropolitan Connecticut areas, 1998. I Infect Dis 2001; 184:1029-1034.

\section{Low-Temperature Hydrogen Peroxide Plasma Sterilization of Bronchoscopes}

Bar and colleagues reported on a study to explore methods to prevent transmission of tuberculosis by bronchoscopes. Bronchoscopes were contaminated with Mycobacterium tuberculosis and decontaminated with a washer-disinfector (normal washing). Some were additionally disinfected with glutaraldehyde (intensive washing). Afterward, the bronchoscopes were sterilized using lowtemperature hydrogen peroxide plasma sterilization.

After normal washing, 8 of 17 samples had positive results by culture, and 7 of 17 had positive results by nucleic acid amplification technique. After intensive washing, all samples had negative results by culture, and 10 of 25 had positive results by the nucleic acid amplification technique. After sterilization with low-temperature hydrogen peroxide plasma sterilization, all samples had negative results by culture and the nucleic acid amplification technique.

The authors concluded that washing of bronchoscopes, as performed normally, is not sufficient for decontamination of bronchoscopes. Additional disinfection is recommended. If the nucleic acid amplification technique is used for diagnostic procedures, sterilization by low-temperature hydrogen peroxide plasma sterilization is recommended to avoid false-positive results.

FROM: Bar W, Marquez De Bar G, Naumann A, Rusch-Gerdes S. Contamination of bronchoscopes with Mycobacterium tuberculosis and successful sterilization by low-temperature hydrogen peroxide plasma sterilization. Am J Infect Control 2001;29:306-311.

\section{FDA Issues Draft Guidance on Blood- Donor Deferrals}

In an effort to reduce the risk of the human variant of mad cow disease, known as Creutzfeldt Jakob disease, the FDA recently issued a 35-page final guidance entitled "Revised Preventive Measures to Reduce the Possible Risk of Transmission of Creutzfeldt-Jakob Disease (CJD) and Variant Creutzfeldt-Jakob Disease (vCJD) by Blood and Blood Products." It recommends deferral of donors who 